\title{
General surgery in haemophiliac patients
}

Elias Bastounis, Emmanouil Pikoulis, Ari Leppäniemi, Dimitrios Alexiou, Christos Tsigris, Andreas Tsetis

\begin{abstract}
With improvements in medical technology, more and larger surgical procedures are performed in haemophiliac patients, but rarely reported in the surgical literature. A retrospective study from a 10 year period from one referral centre identified a total of 68 operations performed in haemophiliac patients. The levels of the defective factors were carefully monitored preoperatively and postoperatively, and replaced according to a standard formula. Special caution was taken to avoid any postoperative medication or procedures that could provoke haemorrhagic complications.

Two patients suffered postoperative bleeding complications that were managed conservatively. Inhibitory factors were detected preoperatively in one case, and postoperatively in another, and were managed with aggressive replacement therapy. There was no mortality, and the overall morbidity rate was $6 \%$.

With adequate preoperative and postoperative monitoring of the clotting factors, meticulous haemostasis during surgery, careful postoperative nursing, and timely replacement therapy, haemophiliac patients can be operated with good results. Postoperative bleeding complications are rare, and usually amenable to conservative management.

(Postgrad Med f 2000;76:494-495)
\end{abstract}

Keywords: surgery; haemophilia; factor VIII concentrates; von Willebrand's disease

A better understanding of the clotting mechanisms and the ability to manufacture factor concentrates have enabled more and larger surgical procedures to be performed in haemophiliac patients. This study reviews the experience of surgical treatment of haemophiliac patients managed in one referral hospital with

1st Surgical

Department,

University of Athens,

Athens, Greece

E Bastounis

E Pikoulis

A Leppäniemi

D Alexiou

C Tsigris

A Tsetis

Correspondence and reprint requests to: Dr Emmanoui Pikoulis, Artemidos 28 Str, Paleo Faliro, 17561 Athens, Greece

Submitted 16 December 1998

Accepted 13 January 2000 special experience in haematological problems.

\section{Patients and methods}

During a 10 year period, from 1985 to 1994, a total of 65 haemophiliac patients underwent surgical treatment in the 1st Surgical Department of the University of Athens. Their mean (range) age was $42(8-72)$ years, and 62 (95\%) were male. Three patients were operated twice for different indications. Forty three patients (66\%) had haemophilia A, 15 (23\%) had haemophilia $\mathrm{B}$, and seven patients $(11 \%)$ had von Willebrand's disease. The type of surgical procedures performed are listed in table 1 .

Before operation, laboratory tests were performed to determine the severity of the dis- ease, the possible presence of inhibitory factors to factor VIII and IX, as well as antierythrocyte antibodies. In addition, liver function tests were performed. The levels of factor deficiencies in the different disease groups are presented in table 2 .

The replacement therapy was conducted immediately before the operation, and the dose required was calculated using the following formula: weight $(\mathrm{kg}) \times$ desired factor level $(\%)$ $\times$ 0.5. Twenty minutes after replacement therapy, the factor level was rechecked, and additional doses of the missing factor were given intraoperatively, as needed.

At operation, the aim was to achieve meticulous haemostasis using ligation for all visible bleeding and cautery only for capillary bleeding, and also trying to keep the operative time to a minimum. Creation of closed spaces was avoided and pressure bandages were used in patients with soft tissue injuries.

Postoperatively, replacement therapy was continued every eight hours for haemophilia A, and every 12 hours for haemophilia B, with daily blood measurements to maintain the factor level above $30 \%$ for $2-3$ weeks. The interval and dose of the postoperative replacement therapy were similar in patients undergoing major or minor surgery for the first three days but the interval was longer, the dose smaller, and the duration shorter in the latter group during subsequent days. Analgesic drugs affecting the clotting mechanism and unneces-

Table 1 Surgical procedures performed in haemophiliac patients

\begin{tabular}{lc}
\hline Surgical procedure & No of patients \\
\hline Abdominal & \\
Inguinal herniorrhaphy & 16 \\
Gastrectomy & 14 \\
Cholecystectomy & 7 \\
Appendicectomy & 3 \\
Hemicolectomy & 2 \\
Splenectomy & 2 \\
Miscellaneous & $4^{\star}$ \\
Excision or incision of cysts and abscesses & 8 \\
Pilonidal cyst & 5 \\
Perianal abscess or fistula & 1 \\
Thyroglossal duct cyst & \\
Extremity procedure & 4 \\
Wound excision for severe soft tissue injury & 1 \\
Above knee amputation & 1 \\
Excision of a soft tissue tumor & 68 \\
Total &
\end{tabular}

*Vagotomy and pyloroplasty, small bowel resection, adhesiolysis, ventral hernia repair.

Table 2 The severity (factor level as \% of normal) of factor deficiency in 65 hemophiliac patients

\begin{tabular}{llll}
\hline Severity & \multicolumn{3}{c}{$\begin{array}{l}\text { Haemophilia Haemophilia von Willebrand's } \\
A(n=43)\end{array}$} \\
\hline Severe $(0 \%-2 \%)$ & 16 & 9 & - \\
Moderate $(2 \%-10 \%)$ & 11 & 3 & 3 \\
Mild $(10 \%-20 \%)$ & 13 & 1 & 3 \\
Latent $(>21 \%)$ & 3 & 2 & 1 \\
\hline
\end{tabular}


sary moving of the patients were avoided. The bandages were changed only when necessary, and after supplying the missing factor. Sutures were removed after 2-3 weeks, and always after factor replacement.

\section{Results}

Four patients had postoperative complications. The first patient underwent a cholecystectomy complicated by intraoperative haemorrhage from the gall bladder bed which continued for 12 days. Blood and fresh frozen plasma were given intraoperatively and postoperatively combined with factor replacement therapy, and the patient was discharged, although he had to be reoperated on three years later for obstructive common bile duct stone in another hospital.

The second patient developed a haematoma after wound excision for soft tissue injury and was treated successfully non-operatively with blood transfusions and replacement therapy.

The third patient who had undergone subtotal gastrectomy two years before for gastric haemorrhage underwent a total gastrectomy for Zollinger-Ellison syndrome, and developed a postoperative subphrenic abscess managed successfully with percutaneous drainage.

The fourth patient underwent a successful cholecystectomy and was discovered postoperatively to have an inhibitor to factor VIII, which was managed with additional replacement therapy using aPCC (Autoplex) with no further complications. In addition, in one patient an inhibitor to factor VIII was detected preoperatively and managed successfully by inducing supranormal levels of clotting factors with aggressive replacement therapy.

\section{Discussion}

Until the 1960s, haemophilia patients underwent only emergency surgical treatment and there were high mortality rates of $25 \%-50 \%{ }^{1}$ After the introduction of the use of part I of plasma (fraction I) in 1960, the mortality decreased below $10 \%$, and further below $2 \%$ with the use of cryoprecipitate. ${ }^{2}$ After the development of a highly active factor VIII and IX concentrates, the mortality rate fell below $1 \%$, and enabled the scheduling of increasingly complex elective cardiovascular and neurosurgical procedures. ${ }^{3}$

In haemophiliac patients, the reported postoperative bleeding complication rate is $3 \%-$ $6 \%$, and the postoperative infection rate about $1 \%,{ }^{4}$ confirmed also in this study.

One of the complications in patients with haemophilia is the development of antibodies

\section{Learning points}

- Adequate preoperative and postoperative monitoring of the clotting factors.

- Meticulous haemostasis during surgery.

- Careful postoperative nursing.

- Timely replacement therapy.

or inhibitors, which have been demonstrated in $8 \%-20 \%$ of patients with severe haemophilia A and in $2.5 \%-16 \%$ of patients with severe hemophilia $\mathrm{B} .{ }^{56}$ Inhibitors develop usually at an early age after few infusions of factor VIII or factor IX, and the treatment of these patients aims at controlling episodes of severe acute bleeding, preparing patients for essential surgery, and to induce immune tolerance by elimination of the antibodies. ${ }^{6}$ In our study, only one patient produced inhibitory factors postoperatively, and was managed successfully with additional replacement therapy. In one of our patients with history of gastric bleeding, high levels of inhibitor factor VIII were detected preoperatively and managed successfully with augmented replacement therapy. In general, if the patient has an inhibitor to factor VIII or IX, we have used either FVIII porcine or aPCC (Autoplex), although during the last two years in patients with inhibitors as well as in patients with acquired hemophilia, we have used recombinant FVIIa with very encouraging results. However, in cases where the risk of bleeding is clearly increased, the postponement of the elective procedure has been suggested. ${ }^{7}$ Postoperative thrombophlebitis has been reported in the literature, ${ }^{7}$ but was not detected in this series. In addition, there were no cases of allergic reactions to the infused factor, or haemolytic reactions caused by antierythrocyte antibodies.

\footnotetext{
1 Craddock CG Jr, Fenniger LD, Simmons B. Hemophilia problem of surgical interventions for accompanying diseases: review of the literature and a report of case. Ann Surg 1980;128:888-904.

2 Pudowski WJ. Major surgery in haemophilia. Ann R Coll Surg Engl 1981;63:111-17.

3 George JN, Brickenbridge RT. The use of factor VIII and factor IX concentrates during surgery. $¥ A M A$ 1970;214: factor IX

4 Rudauski WJ, Schorf R, Ziemski JM. Is major surgery in hemophiliac patients safe? World f Surg 1987;11:378-86.

5 Shapiro SS, Hultin M. Acquired inhibitors to the blood coagulation factors. Semin Thromb Hemost 1975;1:336.

6 Nilsson IM. Factors VIII/IX andibodies. In: Nilsson IM, ed. Hemophilia. Pharmacia Plasma Products, 1994: 58.

7 Kasper CK, Boylen AC, Ewing NP, et al. Haematologic management of hemophilia after surgery. $¥ A M A 1985 ; 253$ : 1279-83.
} 